\title{
Uso del debate como herramienta metodológica docente en estudios del Grado en Medicina: DEBATMITAL
}

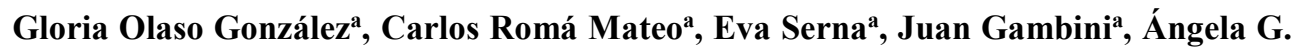
Correas $^{\mathrm{a}}$, Lucía Gimeno ${ }^{\mathrm{a}}$, Consuelo Escriváa ${ }^{\mathrm{a}}$ y Marta Piqueras ${ }^{\mathrm{a}}$.

a Departamento de Fisiología, Facultad de Medicina y Odontología, Universidad de Valencia, gloria.olaso@uv.es

\begin{abstract}
The main purpose of the DEBATMITAL project is to increase the participation of students in the practical session on "Myths of Food" that is part of the optional subject "Nutrition and Dietetics" of the degree in Medicine of the University of Valencia. In front of the master class, which was basically the tool used in previous courses, students are organized into discussion groups to debate some food-related statements commonly heard. The assessment that students make of this initiative is very positive. They indicate that they have learned, they have had fun while participating in the debates, and that they prefer this tool to the master class. Therefore, the debate is a teaching tool that can not only be used in Social Sciences area, but can also be very useful for certain topics in Health Sciences, as in the case of the degree in Medicine.
\end{abstract}

Keywords: methodology, participation, motivation, debate, medicine, nutrition.

\footnotetext{
Resumen

El proyecto DEBATMITAL tiene como objetivo principal aumentar la participación de los alumnos en la sesión práctica sobre "Mitos de la Alimentación" que forma parte de la asignatura optativa "Alimentación y Dietética" del grado en Medicina de la Universidad de Valencia. Frente a la clase magistral, que era básicamente la herramienta utilizada en cursos anteriores, se organiza la clase en grupos de debate para tratar diversas afirmaciones relacionadas con la alimentación que se pueden escuchar comúnmente. La valoración que los estudiantes hacen de esta iniciativa es muy positiva. Indican que han aprendido, que se han divertido mientras participaban en los debates, y que prefieren esta herramienta a la clase magistral. Por tanto, el debate es una herramienta docente que no solo se puede utilizar en el área de las Ciencias Sociales, sino que también puede ser muy útil para determinados temas en grados del área de Ciencias de la Salud, como es el caso del grado en Medicina.
}

Palabras clave: metodología, participación, motivación, debate, medicina, alimentación. 


\section{Introducción}

La asignatura optativa "Alimentación y Dietética" (34493) del grado en medicina de la Universidad de Valencia se oferta en el tercer año de la carrera. Debido a la creciente concienciación sobre la importancia que tiene una alimentación correcta y equilibrada para la salud, la asignatura, desde su implantación, mantiene todos los cursos un número considerable de matriculados (siempre próximo a los 80 alumnos) y además, es una de las más demandadas por los estudiantes de la Nau Gran de la Universidad.

La asignatura tiene una gran carga práctica. Una de las actividades que se realiza consiste en la identificación de mitos en la alimentación entre las afirmaciones que están siendo tradicionalmente transmitidas por el saber popular o incluso en los medios de comunicación. Hasta ahora, esta sesión práctica se llevaba a cabo dando unos minutos para que el alumnado leyera un listado de afirmaciones sobre alimentación y a continuación el profesor o profesora explicaba qué evidencia científica había detrás de cada una de ellas, y cuáles eran fundamentalmente falsas.

Cada curso académico los profesores de la asignatura acusaban que la participación de los estudiantes en esta práctica era escasa, y la mayoría de los alumnos o no asimilaban el mensaje o bien eran reticentes a él (debido al bombardeo en los medios de comunicación reforzando el mensaje contrario). Por tanto, tal y como estaba planteada la práctica se producía una falta de motivación, consecuentemente de participación, y todo ello conducía a una queja cognitiva, puesto que no se debe olvidar que la motivación es uno de los principales motores del aprendizaje (Polanco, 2005) y un determinante del rendimiento académico (Roncel, 2007).

Con la finalidad de aumentar la participación de los estudiantes en esta práctica, se planteó el proyecto DEBATMITAL: El DEBATe como herramienta docente en las prácticas de la asignatura optativa del grado en Medicina "Alimentación y dietética": Identificación de MITos en la ALimentación. Con esta iniciativa se introdujo el debate como herramienta metodológica para trabajar el tema de los mitos en la alimentación. Con el debate se produce un intercambio de ideas sobre un tema determinado y sirve para aproximarse a una situación desde distintos puntos de vista (Esteban, 2017). Es una reconocida técnica de innovación docente que se utiliza especialmente en el ámbito de las Humanidades y de las Ciencias Sociales (Rodríguez-Prieto, 2012). No es una herramienta frecuentemente utilizada en el campo de las Ciencias de la Salud y todavía menos en el caso concreto de la Medicina. Por tanto, la introducción de esta herramienta en las carreras de ciencias es, a día de hoy, bastante original y a través de esta iniciativa hemos podido obtener conocimiento acerca de su utilidad en este tipo de grado. El proyecto DEBATMITAL fue aprobado por la CAT de la Facultad de Medicina de la Universidad de Valencia y financiado a través de la convocatoria de renovación de metodologías docentes de esta misma universidad (UV-SFPIE_RMD18841941). 


\section{Objetivos}

\subsection{Objetivo principal}

El objetivo principal del DEBATMITAL es mejorar la participación de los estudiantes en la sesión práctica sobre mitos de la alientación de la asignatura de Alimentación y Dietética del Grado de Medicina de la Universidad de Valencia.

\subsection{Objetivos secundarios}

Como objetivos secundarios, con el DEBATMITAL se espera fomentar el espíritu crítico de los estudiantes frente a las afirmaciones sobre alimentación socialmente aceptadas. También que se familiaricen con la utilización de la bibliografía científica como fuente de información, así como que mejore su capacidad para trabajar en grupo. Por último, se espera que practiquen la exposición en público y que defiendan su discurso de manera lógica y coherente.

\section{Desarrollo de la innovación}

El proyecto consiste en generar un debate en torno a distintas afirmaciones sobre alimentación que se pueden encontrar de forma habitual en distintos medios de comunicación.

Los estudiantes, dos semanas antes del día programado para la sesión práctica se dividieron en grupos de 6 personas (en total 12 grupos). Se sorteó entre ellos un total de 6 afirmaciones sobre alimentación como por ejemplo: "No desayunar engorda", "La sacarina es cancerígena" o "Comer chocolate hace que salgan granos" (se pueden ver las afirmaciones tratadas en la Tabla 1.) De este modo, se contó con 6 tandas de debate en cada una de las cuales participaban 2 grupos. Uno de ellos defendía la afirmación, y el otro actuaba como abogado del diablo de la misma. En ambos casos, la defensa de cada una de las posiciones debía llevarse a cabo siempre basándose en datos de la literatura científica. El resto de alumnos ejercía de público, pudiendo formular preguntas a los debatientes tras su intervención inicial.

Durante las dos semanas que los alumnos tuvieron para preparar su argumentación, los profesores de prácticas de la asignatura estuvieron a su disposición para cualquier consulta que desearan realizar sobre la búsqueda de la información.

Entre las afirmaciones a debatir algunas eran mitos, otras ciertas y otras presunciones (afirmaciones que se asumen como ciertas pero que no tienen suficiente evidencia científica que las respalde). Hay que señalar que en alimentación nada es blanco ni negro, y que detrás de afirmaciones consideradas como mitos siempre hay alguna pincelada de verdad. Por tanto, aquellos grupos a los que les tocó defender afirmaciones de las clasificadas como mitos, tenían material suficiente en los que poder basar su discurso.

Previamente al comienzo de cada debate, el público (los alumnos restantes) votaba mediante un test realizado a través de la plataforma Kahoot qué opinaban "a priori” sobre la afirmación: si era cierta o si era falsa. 
A continuación, daba comienzo cada debate, moderado por uno de los profesores de prácticas de la asignatura. El equipo defensor de la afirmación y el que estaba en su contra tenían 10 minutos cada uno para exponer sus argumentos. Una vez finalizado este tiempo se daban 10 minutos más para que cada equipo rebatiera las afirmaciones del equipo contrario, así como para atender a las preguntas del público.

Una vez concluidos los 6 debates, se repitió la votación, de nuevo utilizando Kahoot, para ver si la audiencia había cambiado su opinión tras escuchar las argumentaciones de los debatientes.

Tabla 1. Afirmaciones sobre alimentación objeto de debate.

\begin{tabular}{|c|c|}
\hline \multicolumn{2}{|c|}{ ¿MITOS DE LA ALIMENTACIÓN? } \\
\hline M1 & La sacarina es cancerígena \\
\hline M2 & Comer chocolate hace que te salgan granos \\
\hline M3 & El desayuno es la comida más importante del día \\
\hline M4 & El azúcar moreno es más saludable que el blanco \\
\hline M5 & Mezclar hidratos de carbono y proteínas es malo. \\
\hline M6 & Beber agua en las comidas engorda. \\
\hline
\end{tabular}

Hay que puntualizar que el objetivo principal del DEBATMITAL era la mejora de la participación del estudiantado dentro del proceso de aprendizaje y no lo era el ganar el debate. Además, lo que se pretendía era que tras esta sesión práctica los alumnos desarrollaran un espíritu crítico ante cualquier afirmación sobre alimentación que puedan leer u oir en los medios de comunicación o que les llegue a través de la cultura popular, y sepan discernir entre lo que tiene de cierto dicha afirmación y lo que no, como se espera de un profesional de la salud.

Para evaluar el éxito del proyecto, al finalizar la sesión se pasó un cuestionario a los alumnos para que lo cumplimentasen de forma anónima. En las figuras 1 y 2 aparece la imagen de dicho cuestionario. Como se puede apreciar, mediante el cuestionario se pide a los alumnos que valoren la utilidad de la actividad para su aprendizaje, también si les ha resultado entretenida, se les solicita que indiquen cómo se podría mejorar la actividad, y por último si piensan que la actividad les ha ayudado a trabajar algunas de las competencias asociadas a la asignatura y al grado en Medicina. 
Gloria Olaso González, Carlos Romá Mateo, Eva Serna, Juan Gambini, Ángela G. Correas, Lucía Gimeno, Consuelo Escrivá y Marta Piqueras
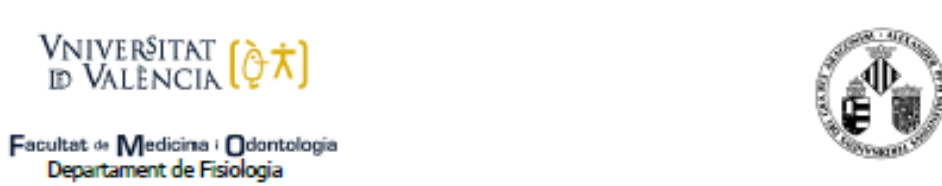

Por favor, imvierte unos pocos minutos de tu tiempo para rellenar el siguiente cuestionario. Valora del 1 al 10 las repuestas, siendo el 1 MUY EN DESACUERDO y el 10 MUY DE ACUERDO.

1. ¿Consideras que gracias a los debates en los que has participado has aprendido?

\begin{tabular}{|l|l|l|l|l|l|l|l|l|l|}
\hline 1 & 2 & 3 & 4 & 5 & 6 & 7 & 8 & 9 & 10 \\
\hline
\end{tabular}

2. ¿la actividad te ha resultado entretenida?

\begin{tabular}{|l|l|l|l|l|l|l|l|l|l|}
\hline 1 & 2 & 3 & 4 & 5 & 6 & 7 & 8 & 9 & 10 \\
\hline
\end{tabular}

3. ¿Consideras que el aprendizaje ha sido mayor que el que puedas adquirir en las prácticas que se realizan siguiendo metodologías docentes clásicas como la clase magistral?

\begin{tabular}{|l|l|l|l|l|l|l|l|l|l|}
\hline 1 & 2 & 3 & 4 & 5 & 6 & 7 & 8 & 9 & 10 \\
\hline
\end{tabular}

4. ¿Qué cambiarías de la actividad?

5. ¿Consideras que la actividad contribupe a desarrollar las siguientes competencizs?

- Comprender e interpretar críticamente textos cientificos.

\begin{tabular}{|l|l|l|l|l|l|l|l|l|l|}
1 & 2 & 3 & 4 & 5 & 6 & 7 & 8 & 9 & 10 \\
\hline
\end{tabular}

Fig 1. Imagen del cuestionario anónimo para la valoración de los alumnos sobre el DEBATMITAL (Parte 1) 
- Tener, en la actividad profesional, un punto de vista crítico, creativo, con escepticismo constructivo y orientado a la investigación.

\begin{tabular}{|l|l|l|l|l|l|l|l|l|l|}
\hline 1 & 2 & 3 & 4 & 5 & 6 & 7 & 8 & 9 & 10 \\
\hline
\end{tabular}

- Capacidad de reunir e interpretar datos relevantes (normalmente dentro de su área de estudio) para emitir juicios que incluyan una reflexión sobre temas relevantes de índole social, científica o ética.

\begin{tabular}{|l|l|l|l|l|l|l|l|l|l|}
\hline 1 & 2 & 3 & 4 & 5 & 6 & 7 & 8 & 9 & 10 \\
\hline
\end{tabular}

- Saber realizar una exposición en público, oral y escrita, de trabajoz científicos $y / 0$ informes profesionales.

\begin{tabular}{|l|l|l|l|l|l|l|l|l|l|}
\hline 1 & 2 & 3 & 4 & 5 & 6 & 7 & 8 & 9 & 10 \\
\hline
\end{tabular}

- Capacidad de transmitir información, ideas, problemas y soluciones a un público tanto especializado como no especializado.

\begin{tabular}{|l|l|l|l|l|l|l|l|l|l|}
\hline 1 & 2 & 3 & 4 & 5 & 6 & 7 & 8 & 9 & 10 \\
\hline
\end{tabular}

- Establecer una buena comunicación interpersonal que capacite para dirigirse con eficiencia y empatía a los pacientes, a los familiares, medios de comunicación y otros profesionales.

\begin{tabular}{|l|l|l|l|l|l|l|l|l|l|}
\hline 1 & 2 & 3 & 4 & 5 & 6 & 7 & 8 & 9 & 10 \\
\hline
\end{tabular}

- Comunicarse de modo efectivo y claro, tanto de forma oral como escrita, con los pacientes, los familiares, los medios de comunicación y otros profesionales.

\begin{tabular}{|l|l|l|l|l|l|l|l|l|l|}
\hline 1 & 2 & 3 & 4 & 5 & 6 & 7 & 8 & 9 & 10 \\
\hline
\end{tabular}

Fig 2. Imagen del cuestionario anónimo para la valoración de los alumnos sobre el DEBATMITAL (Parte II)

\section{Resultados}

La práctica sobre mitos de alimentación se realizó en el salón de grados de la Facultad de Medicina el día 18 de octubre de 2018, cuando la Comunidad Valenciana estaba sufriendo los estragos de la gota fría. Aún así, participaron en la práctica 73 alumnos de los 87 matriculados en la asignatura (alumnos del grado en Medicina y de la Nau Gran). 
A continuación se muestran los resultados obtenidos a partir del DEBATMITAL. Se han agrupado en dos subapartados. El primero hace referencia a la contribución de este proyecto a adquirir los conocimientos de la práctica "Mitos en la Alimentación". En el segundo bloque se muestran los resultados sobre la valoración que hacen los estudiantes del proyecto.

\subsection{Resultados sobre la adquisición de conocimiento.}

Como se ha comentado anteriormente, antes del comienzo de los debates los alumnos opinaban mediante Kahoot si las afirmaciones sobre alimentación a debatir eran verdaderas o falsas. Esas opiniones previas aparecen recogidas en la figura 3. Tras finalizar los debates, se volvió a pedir a los alumnos que valoraran dichas afirmaciones para ver si su opinión había cambiado. (Ver figura 4).

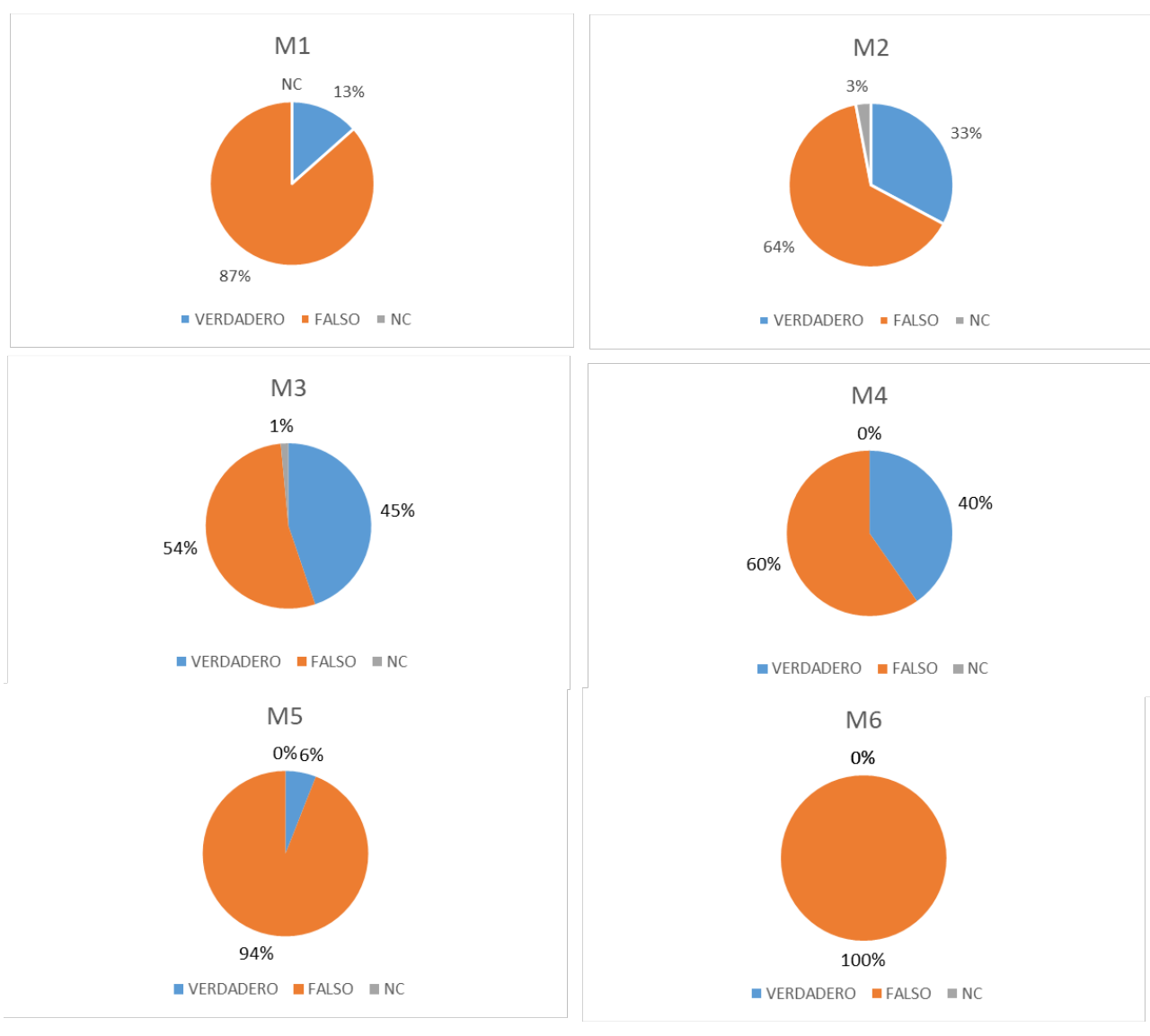

Fig 3. Opiniones previas sobre las distintas afirmaciones trabajadas durante el DEBATMITAL. M1: La sacarina es cancerigena, M2: Comer chocolate hace que te salgan granos, M3: El desayuno es la comida más importante del día, M4: El azúcar moreno es más saludable que el blanco, M5: Mezclar hidratos de carbono y proteínas es malo, M6: Beber agua en las comidas engorda. 


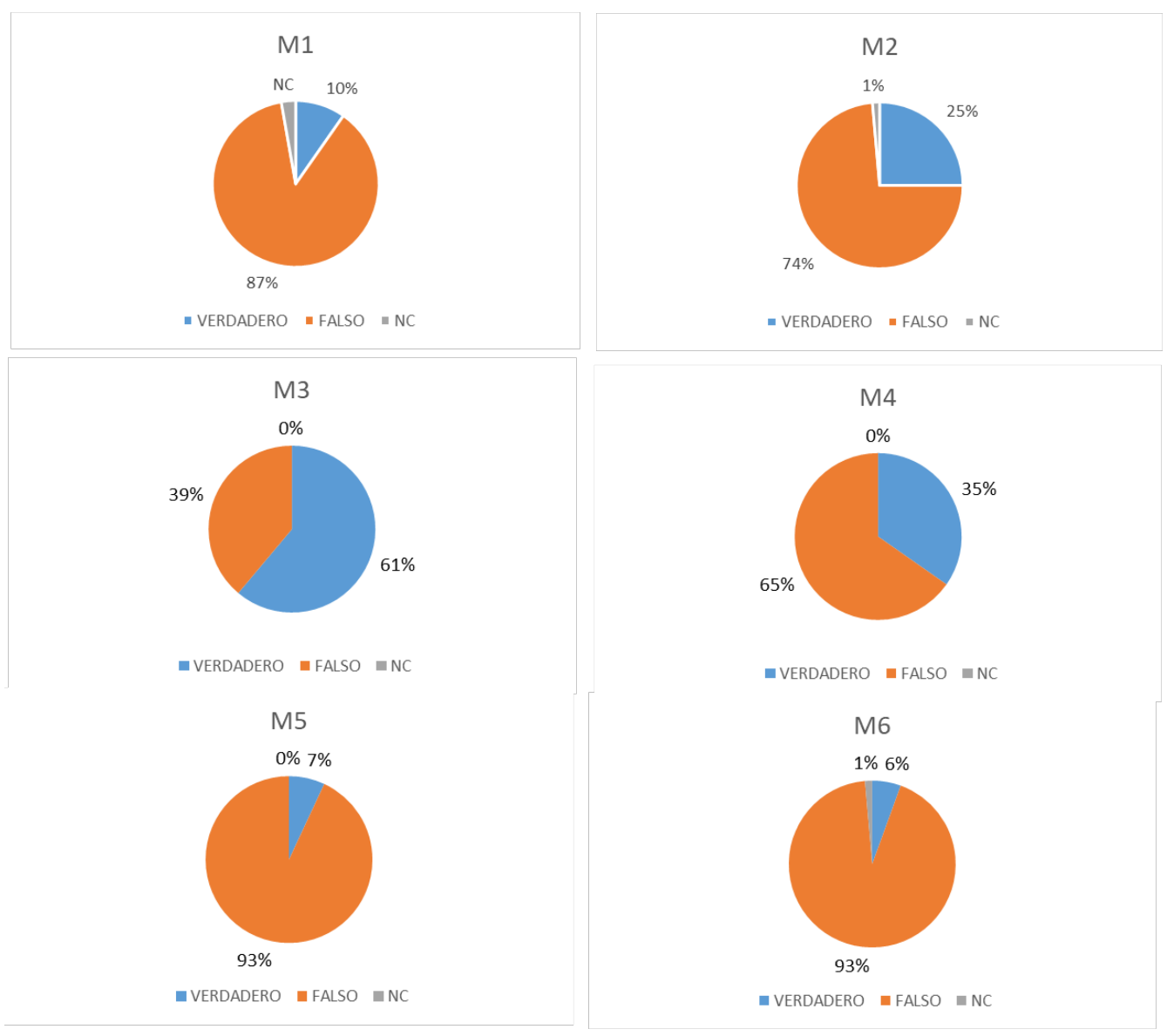

Fig 4. Opiniones posteriores a los debates sobre las distintas afirmaciones trabajadas durante el DEBATMITAL. M1: La sacarina es cancerígena, M2: Comer chocolate hace que te salgan granos, M3: El desayuno es la comida más importante del día, M4: El azúcar moreno es más saludable que el blanco, M5: Mezclar hidratos de carbono y proteinas es malo, M6: Beber agua en las comidas engorda.

Como se desprende de las figuras 3 y 4, la opinión del público (alumnado) no cambia demasiado tras el debate. Los alumnos, previamente a la práctica, ya tenían claro que era verdad y qué era mentira de las afirmaciones a debatir. Son las mismas afirmaciones que se han trabajado desde que se ofrece la asignatura en el grado de Medicina. En el siguiente apartado se aborda con más detalle este tema.

\subsection{Valoración del DEBATMITAL}

Los resultados de la encuesta de valoración del DEBATMITAL contestadas por los alumnos aparecen a continuación. En cada una de ellas, los alumnos debían contestar con un número del 1 al 10. El 1 significaba que estaban totalmente en desacuerdo con lo indicado en la pregunta y el 10 que estaban totalmente de acuerdo.

\subsection{1. ¿Consideras que gracias a los debates en los que has participado has aprendido?}

En la figura 5 se pueden observar los resultados obtenidos para esta pregunta. Como se aprecia, la mayor parte de los alumnos contesta que sí que ha aprendido gracias a los debates. 
De hecho, el $75 \%$ ha puntuado esta respuesta con un valor igual o superior a 8 , siendo la media obtenida para esta pregunta de $8.4 \pm 1.5$ puntos. Según los datos obtenidos, el debate como herramienta docente ha resultado adecuada para el aprendizaje de los alumnos.

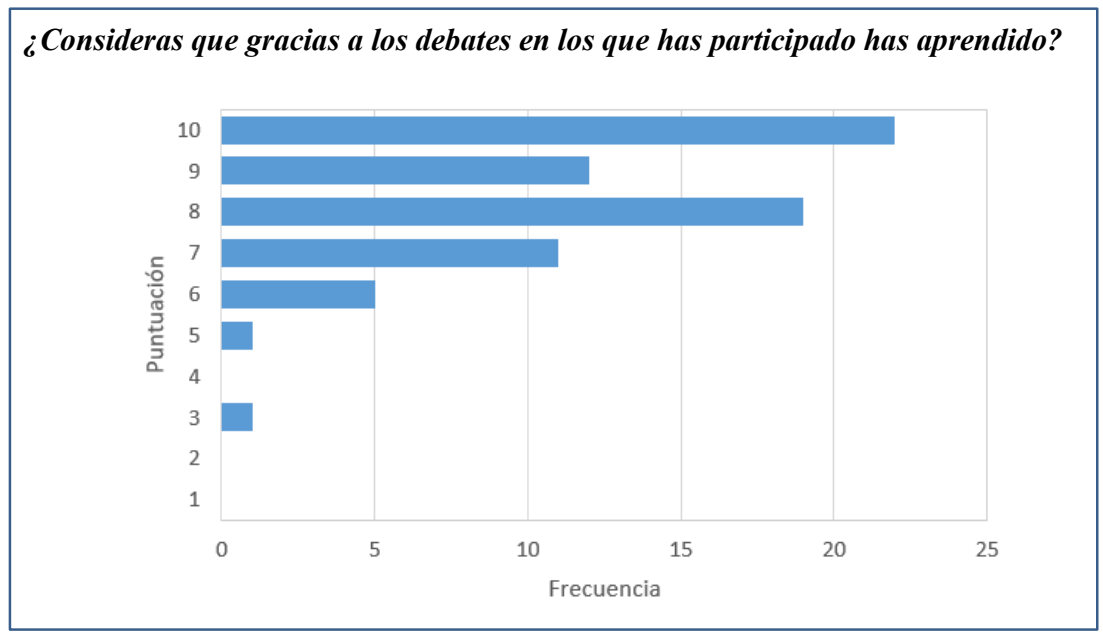

Fig 5. Valoración de los estudiantes a la pregunta ¿Consideras que que gracias a los debates en los que has participado has aprendido? 1: Totalmente de acuerdo. 10: Totalmente en desacuerdo.

\subsection{2. ¿La actividad te ha resultado entretenida?}

Con la pregunta "¿La actividad te ha resultado entretenida?" se pretendía conocer si el aprendizaje (o la falta de él) conseguido gracias al DEBATMITAL se podía relacionar con que la actividad les resultase entretenida o no a los alumnos. Es decir, si el hecho de tener una participación activa en la sesión práctica, les motivaba y contribuía a su aprendizaje.

Las respuestas a esta pregunta aparecen recogidas en la figura 6. A la mayor parte de los alumnos la actividad les ha resultado entretenida. De hecho, la nota media de esta pregunta es de un $8.5 \pm 1.5$, y el $70 \%$ de la clase la ha puntuado con una nota superior o igual a 8 .

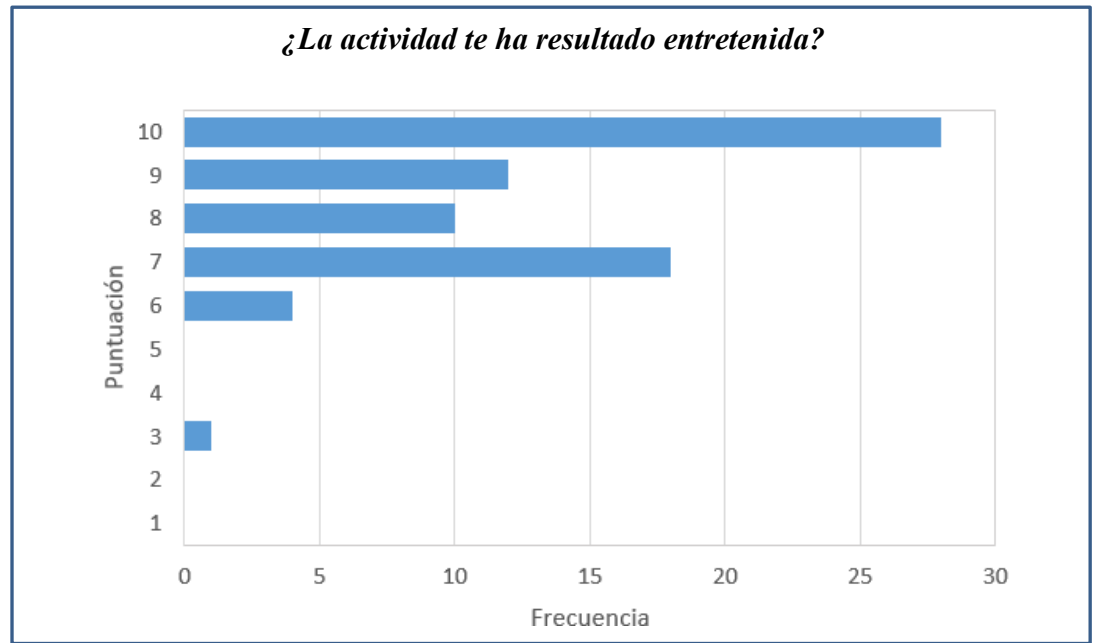

Fig 6. Valoración de los estudiantes a la pregunta ¿La actividad te ha resultado entretenida? 1: Totalmente de acuerdo. 10: Totalmente en desacuerdo. 
4.2.3. ¿Consideras que el aprendizaje ha sido mayor que el que puedas adquirir en las prácticas que se realizan siguiendo metodologías docentes clásicas como la clase magistral?

Queríamos saber si los alumnos preferían este tipo de herramienta docente, mucho más participativa, a la clase magistral que era lo que se había hecho en cursos anteriores para abordar el tema de mitos en la alimentación. La opinión de los alumnos se recoge en la figura 7. De nuevo la puntuación obtenida es de un $8.5 \pm 1.8$. La gran mayoría de los alumnos prefieren esta actividad a la clase magistral. De hecho el $72 \%$ de la clase así lo manifiesta con puntuaciones superiores a 8 . Solo un $3 \%$ de la clase claramente prefiere la clase magistral.

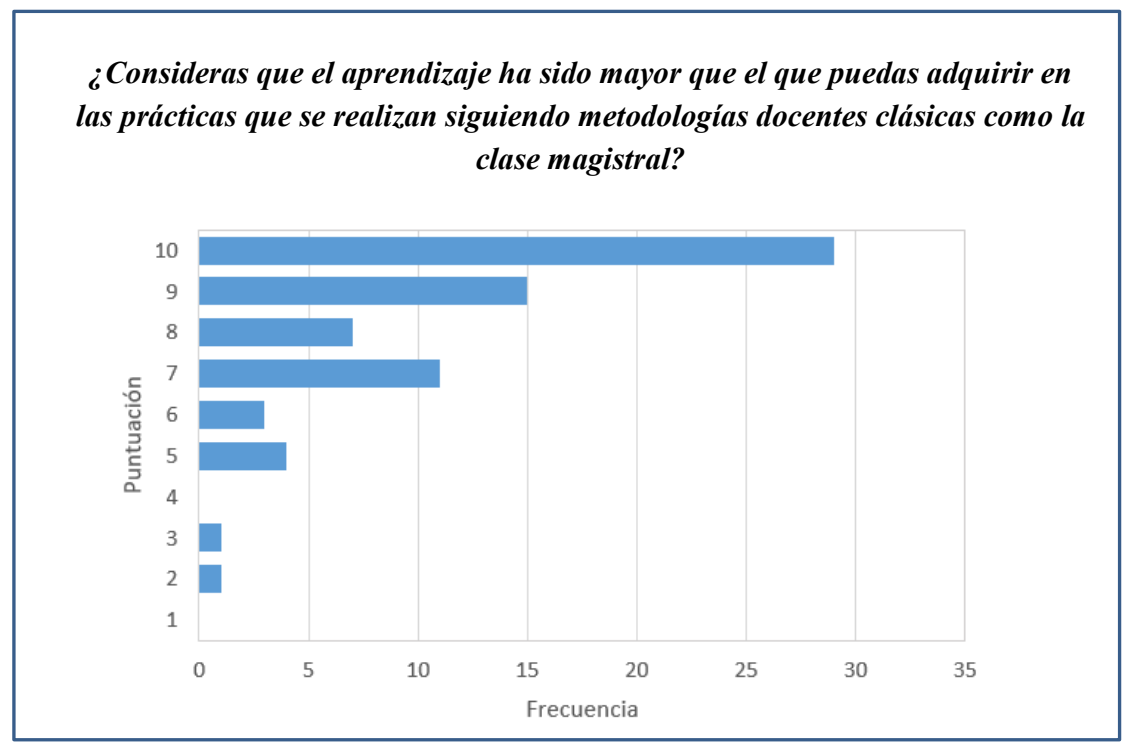

Fig 7. Valoración de los estudiantes a la pregunta ¿Consideras que el aprendizaje ha sido mayor que el que puedas adquirir en las prácticas que se realizan siguiendo metodologías docentes clásicas como la clase magistral? 1: Totalmente de acuerdo. 10: Totalmente en desacuerdo.

\subsection{4. ¿Qué cambiarías de la actividad?}

Esta pregunta abierta permitió a los alumnos expresar de forma no dirigida que cambiarían del proyecto DEBATMITAL. Dos aspectos de la práctica fueron los más comentados. En primer lugar, el tiempo dedicado a cada debate. Varios alumnos han opinado que preferirían que hubiese menos temas de debate y que el tiempo dedicado a cada uno de ellos fuera mayor (cada debate duraba 30 minutos). No obstante, según otras respuestas, algunos alumnos opinaron que los debates eran demasiado largos. Una solución para este aspecto puede ser dedicar varias sesiones para realizar la actividad y compaginarla con algún tema de teoría.

Por otra parte, también varios alumnos opinan que las afirmaciones falsas les resultaban muy evidentes. De hecho, eso se demuestra en que, tal y como hemos comentado previamente, si nos fijamos en las figuras 1 y 2 , tras el debate son pocos los alumnos que han cambiado su postura frente a dichas afirmaciones. Probablemente esto sea debido a que se llevan 
trabajando las mismas afirmaciones desde la instauración del grado en Medicina. De cara al próximo curso académico y se tratarán temas nuevos y más controvertidos.

\subsection{5. ¿Consideras que la actividad contribuye a desarrollar las siguientes competencias?}

Con esta pregunta se quería conocer la opinión de los alumnos sobre la utilidad de la actividad realizada para alcanzar varias de las competencias del grado en medicina. La puntuación conseguida para cada una de ellas aparece en la Tabla 2.

Tabla 2. Valoración de la utilidad del DEBATMITAL para alcanzar competencias del grado en Medicina.

\begin{tabular}{|l|c|c|}
\hline & Nota media & Desviación estándar \\
\hline C1 & 7,0 & 2,2 \\
\hline C2 & 8,1 & 1,5 \\
\hline C3 & 8,0 & 1,7 \\
\hline C4 & 8,2 & 1,5 \\
\hline C5 & 8,2 & 1,3 \\
\hline C6 & 7,7 & 1,6 \\
\hline C7 & 7,8 & 1,8 \\
\hline
\end{tabular}

C1. Comprender e interpretar críticamente textos cientificos, C2. Tener, en la actividad profesional, un punto de vista crítico, creativo, con escepticismo constructivo y orientado a la investigación, C3. Capacidad de reunir e interpretar datos relevantes (normalmente dentro de su área de estudio) para emitir juicios que incluyan una reflexión sobre temas relevantes de indole social, científica o ética, C4. Saber realizar una exposición en público, oral y escrita, de trabajos cientificos y/o informes profesionales, C5. Capacidad de transmitir información, ideas, problemas y soluciones a un público tanto especializado como no especializado, C6. Establecer una buena comunicación interpersonal que capacite para dirigirse con eficiencia y empatía a los pacientes, a los familiares, medios de comunicación y otros profesionales, C7. Comunicarse de modo efectivo y claro, tanto de forma oral como escrita, con los pacientes, los familiares, los medios de comunicación y otros profesionales.

Como se puede concluir de los resultados, los alumnos sí que opinan que la actividad les ayuda a conseguir varias de las competencias que se espera que adquieran a través del grado en medicina.

\section{Conclusiones}

Los estudiantes perciben esta nueva forma de tratar el tema de los Mitos en la Alimentación como útil y entretenida y la prefieren frente a la clase magistral (que era la herramienta docente utilizada hasta el momento).

No obstante, sugieren cambios interesantes que serán tenidos en cuenta de cara al próximo curso académico. Entre ellas, el tiempo dedicado a cada debate y las afirmaciones a debatir dentro de la práctica.

Por tanto, a la luz de los resultados obtenidos se puede concluir que el debate es una herramienta docente muy útil en el grado de Medicina ya que mejora la participación, motivación y aprendizaje del alumnado. 


\section{Referencias}

ESTEBAN, L. (2017). "El debate como herramienta de aprendizaje” en VIII Jornada de Innovación e Investigación docente. Sevilla. Disponible en :

https://idus.us.es/xmlui/bitstream/handle/11441/64625/Debate $\% 20$ como\%20herramienta\% 20de\%20aprendizaje.pdf?sequence $=1$ [Consulta: 24 de marzo de 2019]

POLANCO, A. (2005). "La motivación en los estudiantes universitarios ». Revista Electrónica «Actualidades Investigativas en Educación». Vol. 5, núm. 2, pp. 1-13 http://www.redalyc.org/articulo.oa?id=44750219 [Consulta : 12 de marzo de 2019]

RODRIGUEZ-PRIETO, R. (2012) "El debate como estrategia de innovación docente. Experiencias en Filosofía del Derecho y Teoría de la Cultura". Revista Electrónica «UPO INNOVA». Vol. 1. [Consulta : 12 de marzo de 2019]

RONCEL VEGA, V. M. (2007). El rendimiento en ELE en la enseñanza reglada: un modelo explicativo. MarcoELE: Revista De Didáctica Español Lengua Extranjera, núm. 5. 